\title{
\begin{tabular}{l|l} 
Mitraries & DSpace@MIT
\end{tabular}
}

\author{
MIT Open Access Articles
}

\section{Revealing the Strain Effect on Radiation Response of Amorphous-Crystalline Cu-Zr Laminate}

The MIT Faculty has made this article openly available. Please share how this access benefits you. Your story matters.

Citation: Jin, Miaomiao and Penghui Cao. "Revealing the Strain Effect on Radiation Response of Amorphous-Crystalline Cu-Zr Laminate." JOM: The Journal of The Minerals, Metals \& Materials Society 72, 2 (December 2019): 868-876. (C) 2019 The Minerals, Metals \& Materials Society

As Published: https://doi.org/10.1007/s11837-019-03932-8

Publisher: Springer Science and Business Media LLC

Persistent URL: https://hdl.handle.net/1721.1/128502

Version: Author's final manuscript: final author's manuscript post peer review, without publisher's formatting or copy editing

Terms of Use: Article is made available in accordance with the publisher's policy and may be subject to US copyright law. Please refer to the publisher's site for terms of use. 


\section{Revealing the Strain Effect on Radiation Response of Amor- phous-Crystalline $\mathrm{Cu}-\mathrm{Zr}$ Laminate}

Cite this article as: Miaomiao Jin and Penghui Cao, Revealing the Strain Effect on Radiation Response of Amorphous-Crystalline Cu-Zr Laminate, JOM https://doi.org/10.1007/s11837019-03932-8

This Author Accepted Manuscript is a PDF file of an unedited peer-reviewed manuscript that has been accepted for publication but has not been copyedited or corrected. The official version of record that is published in the journal is kept up to date and so may therefore differ from this version.

Terms of use and reuse: academic research for non-commercial purposes, see here for full terms. https://www.springer.com/aam-terms-v1 


\title{
Revealing the strain effect on radiation response of amorphous-crystalline $\mathrm{Cu}-\mathrm{Zr}$ laminate
}

\author{
Miaomiao Jin* \\ Department of Nuclear Science and Engineering, \\ Massachusetts Institute of Technology, \\ 77 Massachusetts Avenue, Cambridge, MA 02139, USA \\ Penghui Cao \\ Department of Mechanical and Aerospace Engineering, \\ University of California, Irvine, Irvine, CA 92697, US
}

\begin{abstract}
Nanocrystalline materials containing amorphous intergranular films (AIFs) exhibit excellent mechanical properties, radiation resistance, and thermal stability, which may serve as promising candidate materials in advanced nuclear energy systems. Here, we aim to unveil the effect of mechanical stress on the radiation damage behavior of the AIF systems. Based on a bi-crystal Cu system with Zr-doped AIFs, we use molecular dynamics to simulate the radiation process, and examine the AIF sink efficiency, defect propensity, defect size distribution, and Zr mixing under uni-axial and hydrostatic strain conditions. We found the sink efficiency of the glue-like AIFs is not compromised with the applied strains. The anisotropy resulting from the intrinsic microstructure and elastic deformation leads to distinct radiation response, where extension (contraction) of the structure perpendicular to AIFs increases (decreases) vacancy density. The strain dependent defect density, along with the cluster size distributions can be interpreted from variations in defect formation energy and anisotropic defect diffusion. Finally, the Zr mixing induced by collision cascades is found insensitive to the mechanical strains. These findings provide meaningful information towards understanding the stress effect on the radiation response of AIF systems.
\end{abstract}

Keywords: Nanocrystalline, amorphous intergranular film, radiation damage, strain effect, molecular dynamics

\footnotetext{
*mmjin@mit.edu (Corresponding author)
} 


\section{INTRODUCTION}

Screening of radiation-resistant structural materials for nuclear applications prioritizes material self-healing capability under intense and prolonged radiation, because radiationinduced responses such as embrittlement, swelling, creep, and hardening can significantly degrade mechanical properties and dimensional integrity [1]. Nanocrystalline (NC) materials with a large amount of interfacial areas as defect sinks suggest a promising solution, however, they are usually brittle due to constraint of dislocation motion across grains and they also demonstrate high grain growth rate, which can be largely accelerated by radiation $[2,3]$. To resolve this issue, interface engineering which manufactures amorphous intergranular films (AIFs) instead of conventional grain boundaries has shown increased mechanical performance and thermal stability $[4,5]$. The potential utilization as structural materials in the harsh environment of future nuclear energy systems exposes an inevitable issue of stress effects on the radiation response.

Radiation assisted microstructure evolution which dictates material performance varies with stress condition. It was suggested that stress can stabilize void nuclei, and shorten the transient swelling regime in 316 stainless steels $[1,6]$. In $\mathrm{SiO} 2 / \mathrm{Si}$ interfacial region, compressive stress was found to reduce both positive charge build-up and interface-trap generation [7]. Dislocation loops were found to form preferentially with their normal aligning with the direction of tensile stress [8], and the loop-punching process in helium bubble growth is tweaked by the external strain field [9]. Although there was some theoretical development in incorporating stress/strain in evolution such as swelling $[1,10]$, the assumptions exclude defect dynamics in the presence of complex structures such as various interfaces and existing defect clusters. Therefore, to resolve the correlation between evolution details and external stress, it is necessary to deliberately construct specific structures on the atomistic scale, apply stress/strain condition, and capture the primary radiation damage process on the scale of tens of pico-seconds which includes the processes of ballistic collision, thermal spike, and annealing.

Simulating the primary damage can be relatively easily performed via molecular dynamics (MD) with high fidelity. It is commonly accomplished by assigning an atom (primary knocked-on atom, PKA) with a prescribed kinetic energy, and atom collisions occur within a large enough simulation cell. Then the system anneals to the ambient temperature in about 
tens of pico-seconds. By applying various strains on the simulation system, the influence on defect production and clustering can be examined. For example, Beeler et al. quantified the defect generation from a $5 \mathrm{keV}$ PKA in Fe, and concluded that strains with volume changes can produce significant effects while volume-conserving shear strains yield insignificant variations [11]. More generally, depending on the material system, the number of generated defects from a single PKA can increase or decrease upon different strain directions $[12,13]$. This strain effect extends to the size of defect clusters $[12,14]$. Additional defect property regarding threshold displacement energy [15, 16], formation [17] and migration energy [18] can also be modified.

An AIF system inherently differs from conventional steels due to its unique internal structures. Thus the stress-affected radiation response is of scientific interest and practical concern. Here, we study NC Cu with a bi-crystal system. The $\Sigma 5(210)$ grain boundaries (GBs) are transformed into AIFs with Zr dopant, which is a simplified model to the experimental samples [4]. Under different strain conditions (uni-axial and hydrostatic), multiple aspects are investigated with MD simulations to reveal stress effect, including AIF sink efficiency, defect evolution, and Zr mixing. These results suggest that a directional stress dependence of radiation response, and this AIF engineering can be optimized by introducing predominate boundary directions with respect to the stress condition. Moreover, incorporation of stress factors to larger scale simulation models such as rate theory and kinetic Monte Carlo is necessary to represent modified defect dynamics.

\section{METHODS AND MODELS}

MD code, LAMMPS [19] is used throughout this study, and atomic interactions are described by an EAM potential from Borovikov et al. [20]. To accommodate close-distance collisions, the EAM potential is smoothly joined to the Ziegler-Biersack-Littmark (ZBL) repulsive potential [21]. The initial atomic configuration is shown in FIG. 1, with 117,600 atoms and a dimension $11.4 \mathrm{~nm} \times 11.8 \mathrm{~nm} \times 11.0 \mathrm{~nm}$. With periodic boundary condition, the gray atoms indicate the two AIFs. The AIF is created by i) doping $1.0 \mathrm{~nm}$ slice of atoms containing a high angle symmetric tilt $\Sigma 5(210)$ GB with 25 atomic\% Zr atoms; ii) melting the slice at $1600 \mathrm{~K}$ for $200 \mathrm{ps}$; ii) quenching the slice from $1600 \mathrm{~K}$ to $650 \mathrm{~K}$ over 200 ps. Finally, the entire system is quenched from $650 \mathrm{~K}$ to $300 \mathrm{~K}$ over $200 \mathrm{ps}$, and relaxed 
at $300 \mathrm{~K}$ for $30 \mathrm{ps}$ under zero pressure. The final thickness of the AIF is around $1.2 \mathrm{~nm}$.

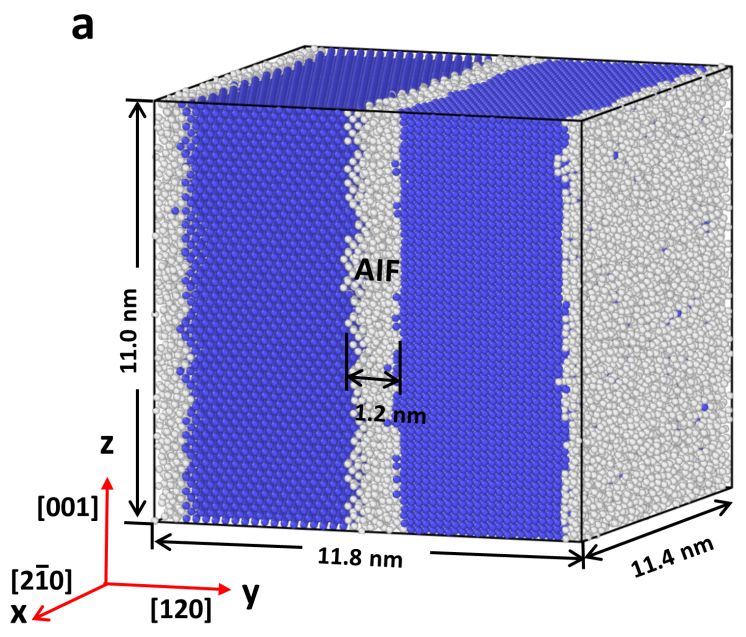

b

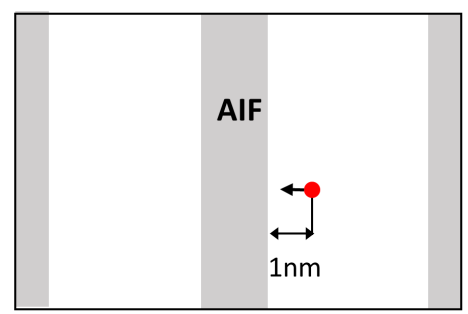

C

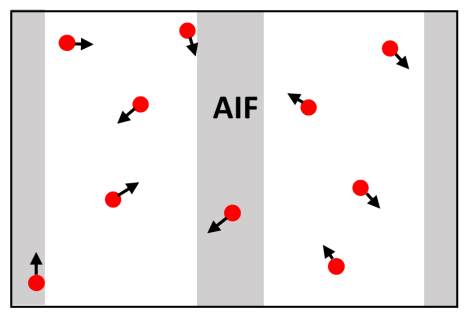

FIG. 1. (a) A bi-crystal simulation model with two AIFs, which are constructed by a heat treatment process of $\Sigma 5(210)$ GBs doped with $\mathrm{Zr}$ atoms ( $\mathrm{Cu}-25$ at.\% $\mathrm{Zr}$ ). The parameters, $\lambda$ and $d$ denote the AIF inter-distance and width, respectively. Atoms are colored by the structure type (blue: FCC atoms, gray: non-FCC atoms). (b) Schematic illustration of a single cascade with a PKA located $1 \mathrm{~nm}$ away from the interface launching towards the interface. (c) Schematic illustration of multi-cascade simulation with consecutive PKAs randomly introduced in the simulation cell.

Uni-axial strain from $-2 \%$ to $2 \%$ and hydrostatic strain from $-2 \%$ to $0.5 \%$ with $0.5 \%$ increment are considered, where positive and negative values indicate tensile and compressive conditions, respectively. It should be noted under high hydrostatic strain $(>0.5 \%)$, the structure is found not stable during irradiation. The strain is applied to the prepared system at $300 \mathrm{~K}$. For the uni-axial strain, the other two dimensions are under zero pressure, so they respond to the strain dynamically. Then the system is relaxed at constant volume at the strain state before damage process. For radiation damage, $5 \mathrm{keV}$ PKAs are utilized, and an adaptive time-stepping technique is used to restrain the maximum moving distance below $0.05 \AA$ per timestep. A thermal bath of $300 \mathrm{~K}$ is created by applying a temperature-rescaling thermostat at all sides to absorb excess energy. Here, we consider two different scenarios. On one hand, to compare the sink efficiency of AIFs under different strain states, the PKA is launched directly towards the AIF at $1.0 \mathrm{~nm}$ distance away from the interface (FIG. 1b), and the residual defects are compared. On the other hand, continuous irradiation is simulated by 
consecutively introducing random PKAs with random incident directions (FIG. 1c). Since the PKA is randomly selected, it is always reset to the center by shifting the simulation cell, to make sure that the damage cascade induced by the PKA is fully contained in the cell. After annealing the system to $300 \mathrm{~K}$ over around $30 \mathrm{ps}$, the cell is shifted back in order to track defect evolution consistently. By increasing the number of PKAs, one can obtain a high dose level based on the estimation with Norgett-Robinson-Torrens (NRT) model. Using this method, the system evolution process can be compared.

Based on the atomic configurations, defects are identified via Wigner-Seitz cell method [22] by comparing with the reference configuration. Defective structures are recognized with common neighbor analysis. Visualization of atoms is generated with the OVITO package [23]. The migration barriers of point defects are calculated using the NEB method [24] as implemented in LAMMPS.

\section{RESULTS}

AIF can be perceived as a separate phase from the FCC bulk [25], which responds differently to external strain. For example, with the prescribed strain $-2 \%$ and $2 \%$ in y-direction, AIF demonstrates softness as shown in Table I. Although AIF thickness, Zr concentration, and lattice orientation of the bulk may affect the specific values, the relative softness of the amorphous phase is expected to maintain. The AIF acts like flexible "glue" between hard bodies, which may weaken the strain effect on radiation response compared with grain boundary structures. The association between strain and the radiation response is elaborated in the following sections regarding the AIF efficiency, defect dynamics, and ion mixing due to damage cascades.

\begin{tabular}{|l|l|l|l|l|}
\hline Prescribed strain $(\%)$ & AIF thickness $(\AA)$ & AIF strain $(\%)$ & Bulk layer thickness $(\AA)$ & Bulk strain $(\%)$ \\
\hline-2.0 & 11.8443 & -2.91 & 45.7992 & -1.76 \\
\hline 2.0 & 12.4929 & 2.41 & 47.5031 & 1.89 \\
\hline 0.0 & 12.1992 & 0.0 & 46.6203 & 0.0 \\
\hline
\end{tabular}

TABLE I. Decomposed strain in AIF and bulk under prescribed strain $-2 \%$ and $2 \%$ in y-direction. 


\section{A. AIF efficiency}

The sink efficiency of AIFs is compared via the number of residual point defects. By simulating the damage cascade induced by a single PKA at $1 \mathrm{~nm}$ near the interface (FIG. 1b), the number of residual defects is counted under all strain conditions. FIG. 2 depicts the average number of vacancies $\left(N_{\text {vac }}\right)$ and interstitials $\left(N_{\text {sia }}\right)$ in the bulk region of 20 systems, which are thermalized at $300 \mathrm{~K}$ with different random numbers. It can be seen the remaining vacancies mostly outnumber interstitials, and from the error bars, $N_{v a c}$ varies more drastically than $N_{\text {sia }}$. The underlying reason is that the temperature effect causes deviation from head-on collisions. As such, generated vacancies are more populated close to the PKA initial location and at this temperature, vacancies are still not mobile enough to sink at the interface, therefore, high $N_{v a c}$ is obtained. On the contrary, $N_{\text {sia }}$ has a lower value and generally narrower error bar, due to the forward scattering toward AIF and higher mobility of interstitials compared to vacancies.

Among all the strain states, $N_{\text {sia }}$ appears consistent at a value around 2-3, and $N_{v a c}$ is higher, around 5. The high sink efficiency remains regardless any strain conditions. Considering the large error margin, there is no statistically significant trend between interface efficiency and strain states. The defect production in perfect crystals relates to the displacement threshold energy $\left(E_{d}\right)$. Tension supposedly decreases $E_{d}$ while compression leads to higher $E_{d}[15]$. However, it should be mentioned that the damage cascades partially overlapping with AIFs leads to the calculation of $E_{d}$ nonessential. More insights can be gained by considering the accumulating consequence of multiple cascades which incorporate the overlapping of damage cascades and the randomness of PKA direction and location.

\section{B. Defect evolution}

Multi-cascade simulations enable a dynamic view of system evolution due to intense radiation, and are especially valuable in studying low temperature radiation where thermal diffusion is neglected. By consecutively introducing random cascades into the strained system, we count the defect population in the sample region as schematically shown in FIG. 3a. As an example, FIG. 3b depicts the vacancy concentration for y-direction strain versus increasing dose. After the initial gradual increase in vacancy density, fast saturation quickly 

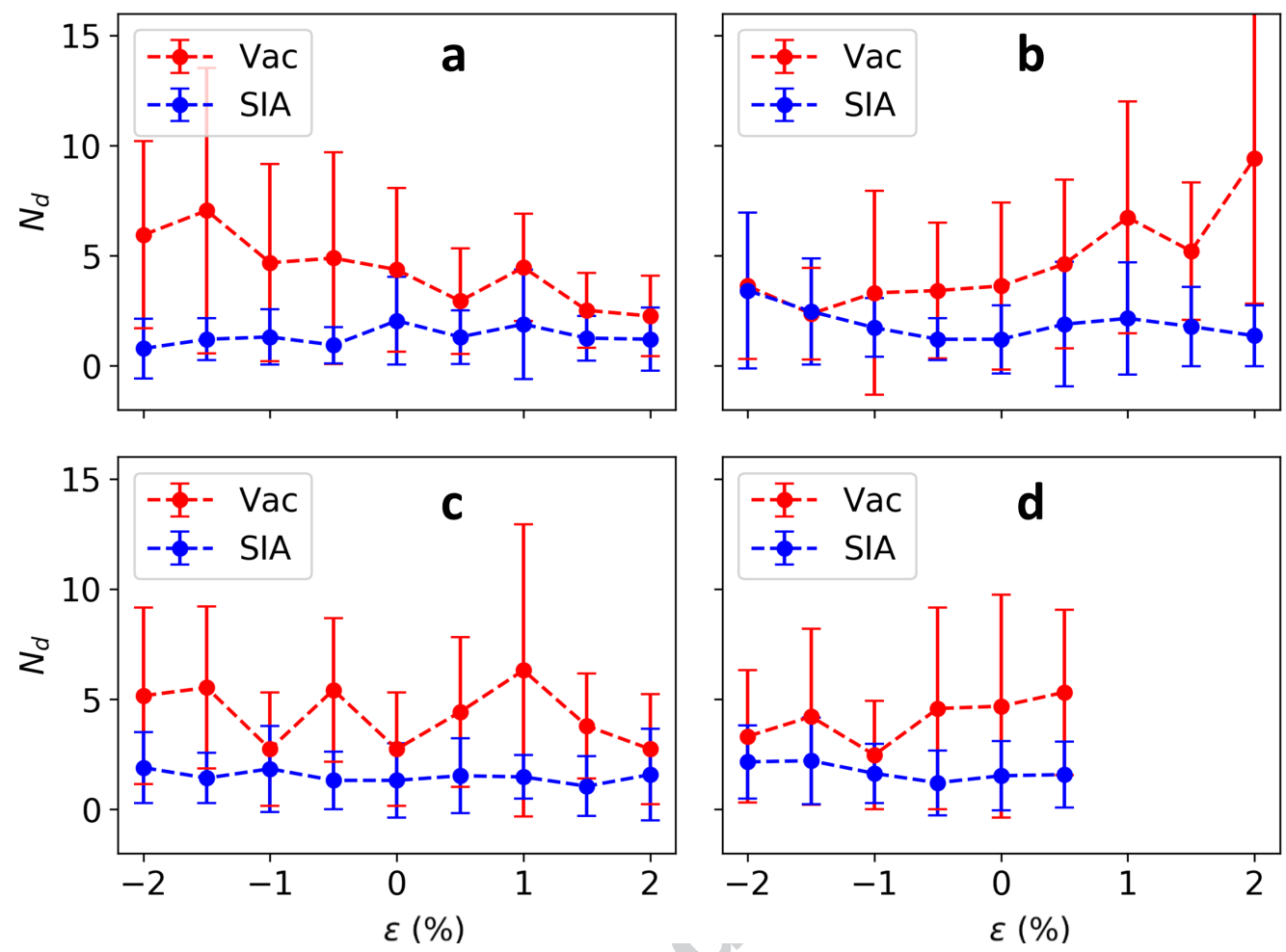

FIG. 2. Number of bulk residual vacancy (Vac, red dot) and interstitial (SIA, blue dot) produced by single cascade simulations for the system applied at different strains. (a), (b), and (c) have the system strained in $\mathrm{x}, \mathrm{y}$, and $\mathrm{z}$, respectively, and (d) in hydrostatic strain. Error bars represent one standard deviation of uncertainty from 20 simulations.

occurs in the system. The same pattern applies to the interstitial density, but due to a much higher mobility of interstitials which contributes to the preferential absorption of interstitials at AIFs, the curves saturate at a much smaller quantity. The saturated values can then be calculated by averaging the corresponding data points for each strain, as shown in FIG. 4. Interstitials are scarce in the bulk region and hence the density maintains small, with 1-2 orders of magnitude lower than that of vacancy. Due to the large standard error, no obvious relation can be drawn between interstitial density and strain (FIG. 4a-d). On the other hand, vacancy density manifest a correlation with strain intensity $(\epsilon)$ depending on the strain direction (FIG. 4e-h). Specifically, for x- and z- strain, vacancy density slightly decreases with $\epsilon$, while for $\mathrm{y}$ - and hydrostatic strain, it increases with $\epsilon$. Quantitatively, compression in y-direction and hydrostatic largely suppress the vacancy density, and vice versa, compared with $\mathrm{x}$ - and z-direction strain conditions. 

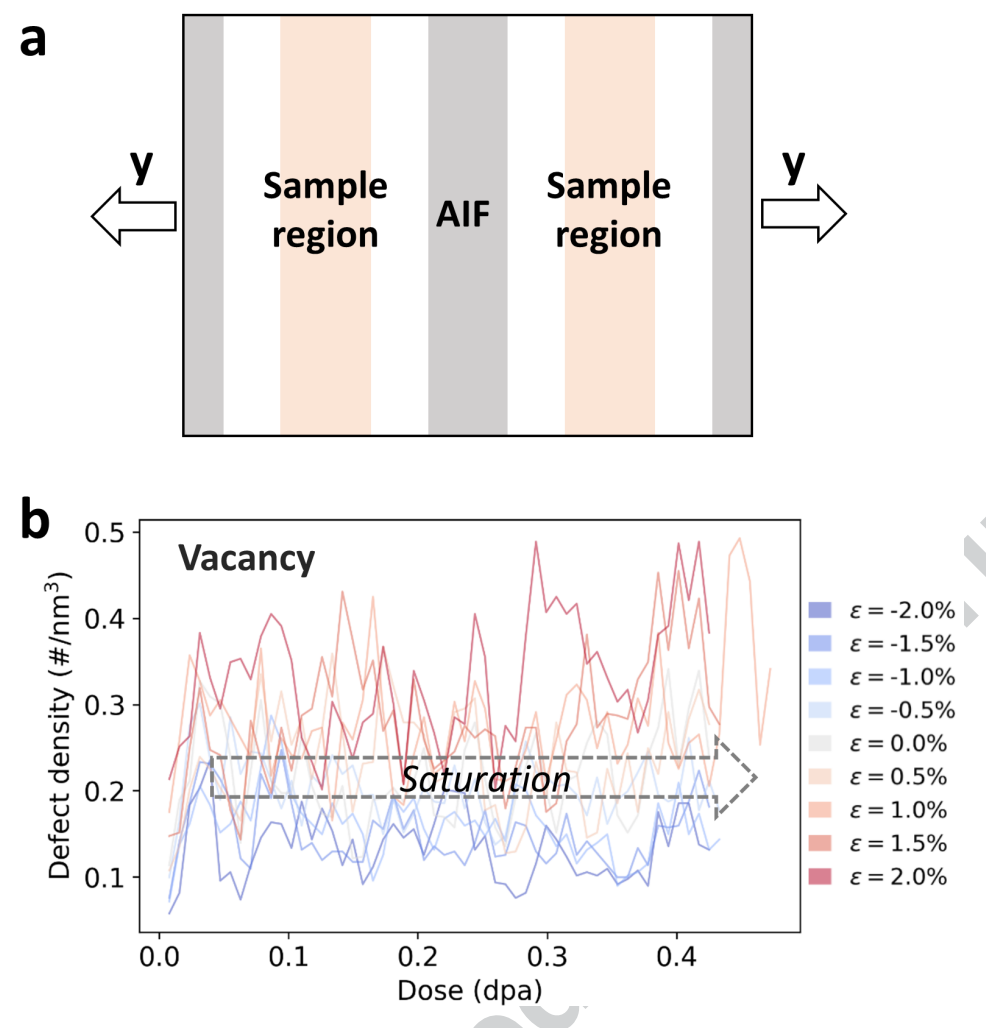

FIG. 3. (a) Schematic demonstration of simulation cell with uni-axial strain applied in y direction. Defects in the "sample region" are counted. (b) Evolution of vacancy density versus dose for various y-direction strain levels, which shows defect saturation.

The defect size distribution can reflect the modified defect dynamics due to strain. FIG. 5 depicts the four scenarios with histograms in binning size 5 . The large sizes of defect clusters are mainly in the form of SFTs (stacking fault tetrahedra), from direct production from damage cascades and vacancy aggregation near the cascade volume. It can been seen that in tension in y-direction significantly increases defect size (FIG. 5b), and compression in $\mathrm{x}$ - and $\mathrm{z}$ - direction also increases defect size. For hydrostatic compression, clusters are generally small.

\section{Ion mixing}

The athermal processes of ballistic collision and thermal spike contribute to the phenomenon called ion mixing, and the latter dominates especially in low energy irradiation [26]. The atomic mixing process can be well described by the diffusion model in various 

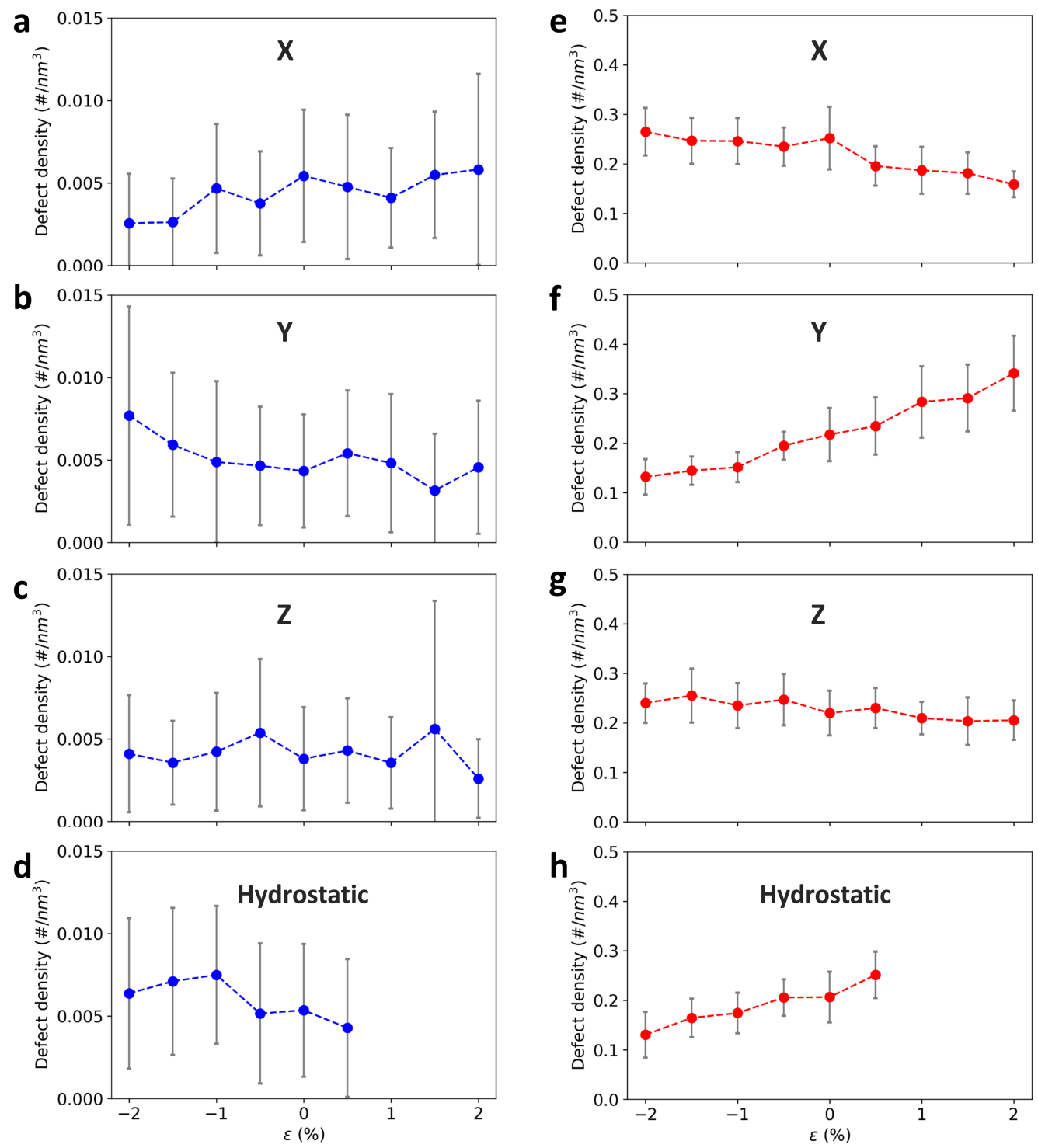

FIG. 4. Saturation density of interstitial (a-d) and vacancy (e-h) for various strain schemes, where 'X', 'Y', 'Z', and 'Hydrostatic' stand for uni-axial x, y, z, and hydrostatic strain, respectively. Each error bar represents one standard deviation of uncertainty based on 35 data points.

bilayer experiments [27]. By fitting to the solution (i.e. error function) of thin film diffusion problem [28], the variance of the distribution profile ( $4 D t$, where $D$ is the effective diffusion coefficient for mixing and $t$ is time) as a measure of mixing level can be calculated at different doses for various strain states. As a demonstration, FIG. 6a depicts Zr distribution at three damage levels $(0.08,0.24$, and $0.40 \mathrm{dpa})$, where the distribution is calculated based on the histogram of the horizontal (i.e. $\mathrm{y}$-) coordinates of $\mathrm{Zr}$ atoms. The red curves indicate 

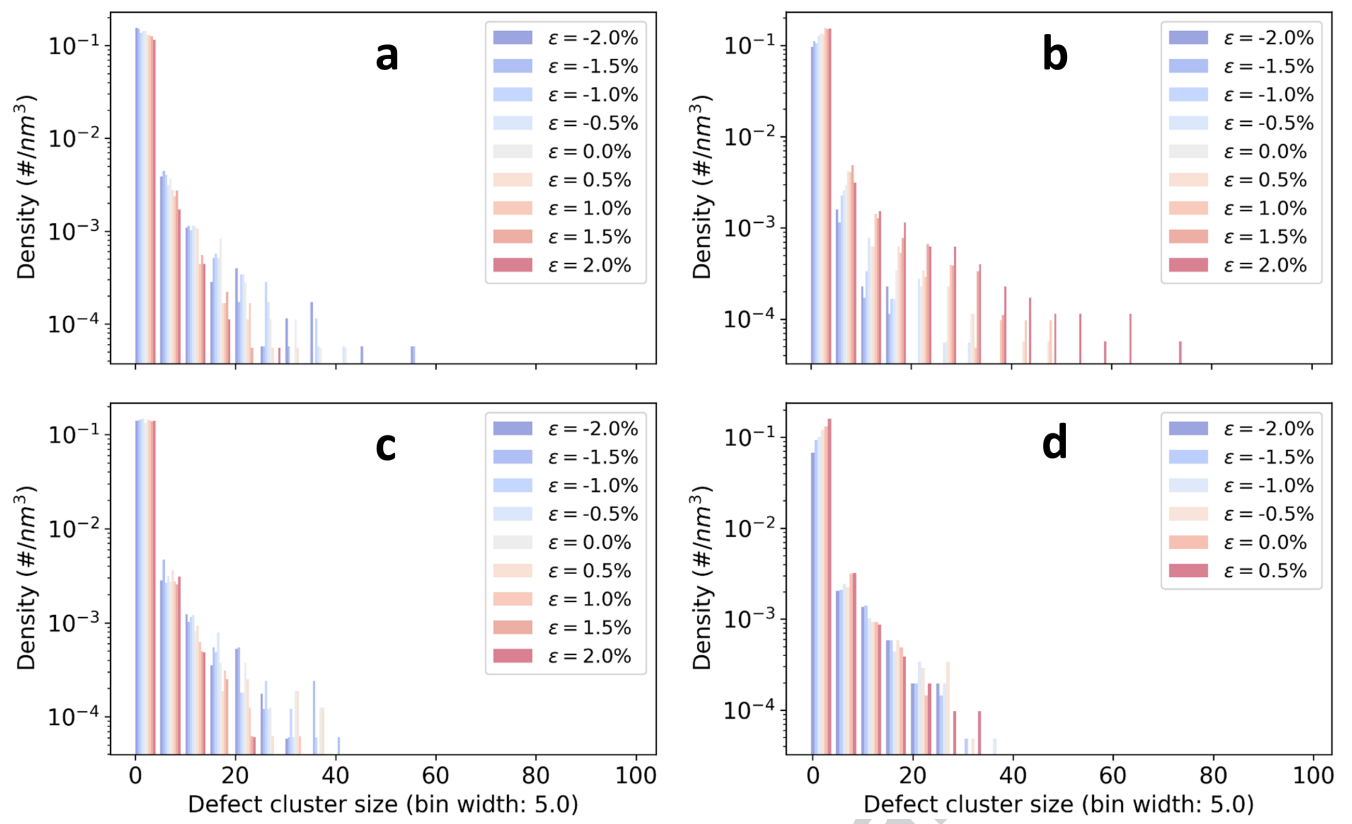

FIG. 5. Defect cluster size distribution for system under strain in $\mathrm{x}(\mathrm{a}), \mathrm{y}(\mathrm{b})$, and $\mathrm{z}$ (c) uni-axial , and hydrostatic (d) directions, respectively. Binning width for histogram is 5.

strong agreement with the histograms after fitting the multilayer diffusion solution. $\sqrt{4 D t}$ is indicated in the relevant figures. For all the strain conditions, we then retrieve $4 D t$ with respect to dose as exemplified in FIG. 6b, and $4 D t$ scales linearly with dose. Similar mixing results are obtained for all other strain conditions. It can be summarized that strain does not exhibit an obvious impact on the mixing behavior during accumulated cascades.

\section{DISCUSSION}

The anisotropy in the AIF-system comes from both the microstructure and deformation. An orientation relationship of the adjacent bulks and AIF are expected to cause distinct responses among the uni-axial strain schemes, let alone the hydrostatic one. The AIFs were perceived as 'glue', strongly increasing the ductility of nanocrystalline materials $[4,5]$. In contrast with the crystalline bulk, the amorphous layer appears more ductile (Table I). For a pure $\mathrm{Cu}$ system without any interfaces, Miyashiro et al. [12] utilized MD simulations and found that defect production rate in cascade increases significantly with both uni-axial tensile and compressive stresses, but not so much under hydrostatic one. However, in current AIF-systems, the existence of interface makes the quantification of defect production less 
a
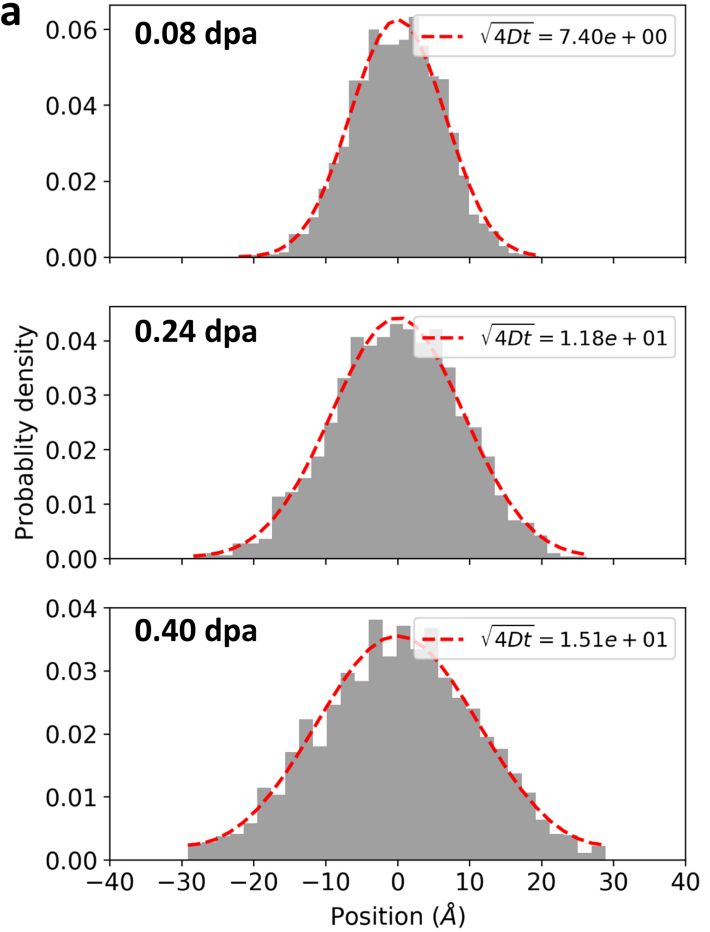

b

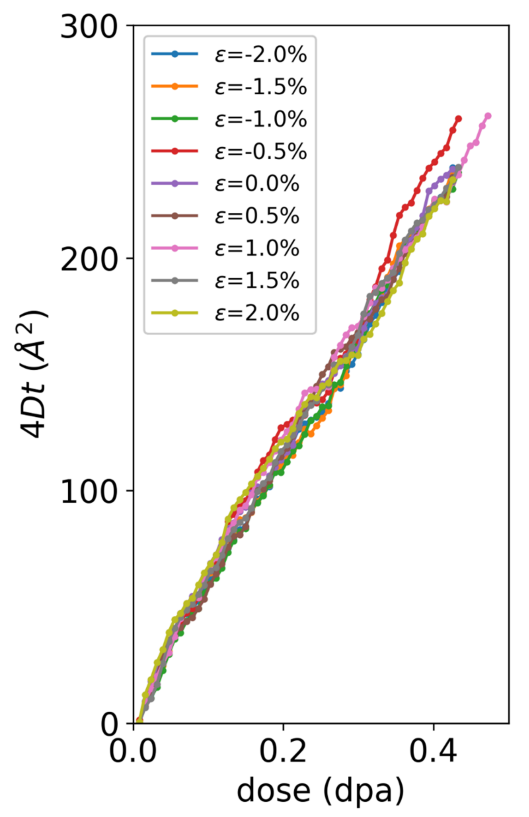

FIG. 6. (a) The distributions of $\mathrm{Zr}$ atoms at three different damage levels as indicated. The red dashed curves plot error function fitted to the histogram and $\sqrt{4 D t}$ is a fitting parameter measuring the spread of distribution. (b) $4 D t$ versus dose for different strain values in y-direction.

straightforward since the drastic difference of point defect mobility means that interstitial would preferentially sink at the interfaces and the bulk defects are vacancy-type dominant. As such, affected by the distance between cascades and interfaces, the commonly calculated average defect production rate doesn't carry as much meaning in presence of interfaces compared with perfect crystalline systems. To overcome this conundrum, we instead compare the interface sink efficiency and the saturated bulk defect density under the various prescribed strain states.

The amorphous nature of the AIF layers implies high defect sink capability due to large excess free volume. Direct overlap between damage cascade and the interface indicates that interstitials are mostly absorbed at the interface while vacancies are on average more populated, and there is no statistically significant variation upon strain. These results contrast with perfect crystalline systems such Fe [11], W [29], and $\mathrm{Cu}$ [12] where the defect production is correlated with the strain condition to certain levels. The atomic shuffling within AIF absorbs nearby interstitials and accommodate partial cascade, as the PKA is 
directed toward the interface. Such characteristic still holds under strain. However, in realistic irradiation condition, where a PKA can be initiated randomly, interstitials can both recombine with vacancies and sink at the interfaces. The statistics of accumulated defects, especially vacancies in the bulk region can reflect the overall AIF sink efficiency under the influence of strain. The saturated vacancy density indicates a strong directional dependence. Comparing all four scenarios, it appears that decreasing y-dimension will reduce vacancy density and vice versa (note that tension in $\mathrm{x}$ - and $\mathrm{z}$ - direction would lead to an increase in y-dimension due to a positive Poisson ratio). In other words, system extension (contraction) perpendicular to AIF leads to increasing (decreasing) vacancy density. In particular, compression in $\mathrm{x}$ - or $\mathrm{z}$ - direction induces extension in $\mathrm{y}$ direction, and the trend of mean vacancy density with respect to strain changes oppositely to that under y-direction and hydrostatic strain. Previous studies on Fe suggested that the volume changes strongly correlate with the defect generation [11], hence, FIG. 7a depicts the volumetric strain. However, although volume as a macroscopic indication of local stress environment and concomitant modified defect dynamics, volume itself doesn't show any phenomenological relationship with defect population.

The bulk defect density and cluster distribution result from a combination of defect production, defect-defect interaction, defect-interface interaction, and overlap between cascades and existing defects. The formation energy and migration barrier of point defect can shed light on the results in FIG. 4 and 5. FIG. 7b-c shows the formation energies of vacancy and interstitial, which are calculated by $E_{f}-\frac{N_{0}-1}{N_{0}} E_{i}$ and $E_{f}-\frac{N_{0}+1}{N_{0}} E_{i}$, respectively. Herein, $E_{f}$ is the total energy of the system with one defect, $N_{0}$ is the initial number of atoms, and $E_{i}$ is the total energy of initial perfect system. Vacancy is simply the deficiency of one lattice atom, while interstitials are in the form of $\langle 100\rangle$ dumbbell. Multiple calculations with different vacancy positions and interstitial orientations are performed to ensure good statistics. We can see that vacancy formation energy is highly positively correlated with the volumetric strain (FIG. 7a). Interstitial formation energy exhibits a steeper opposite trend because of necessary excess volume to accommodate an extra atom. Hence, the formation energy explain well the defect density in the hydrostatic scenario which induces significant volume change. However, discrepancy arises by comparing the uni-axial loadings where the formation energy doesn't vary much. Thus, the migration energies of point defects are calculated to explain from the kinetics' point of view. It should be noted that although the 


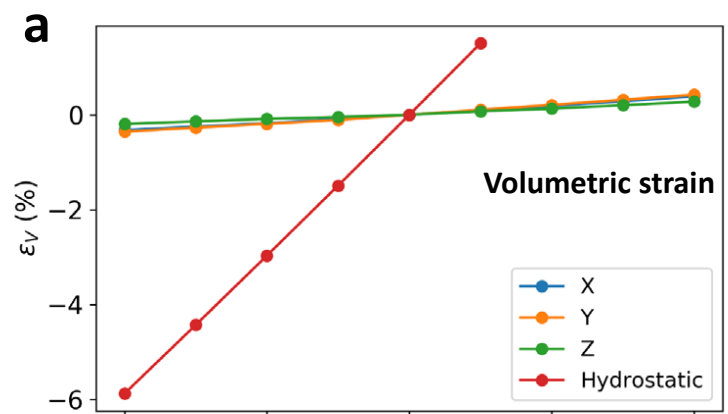

d
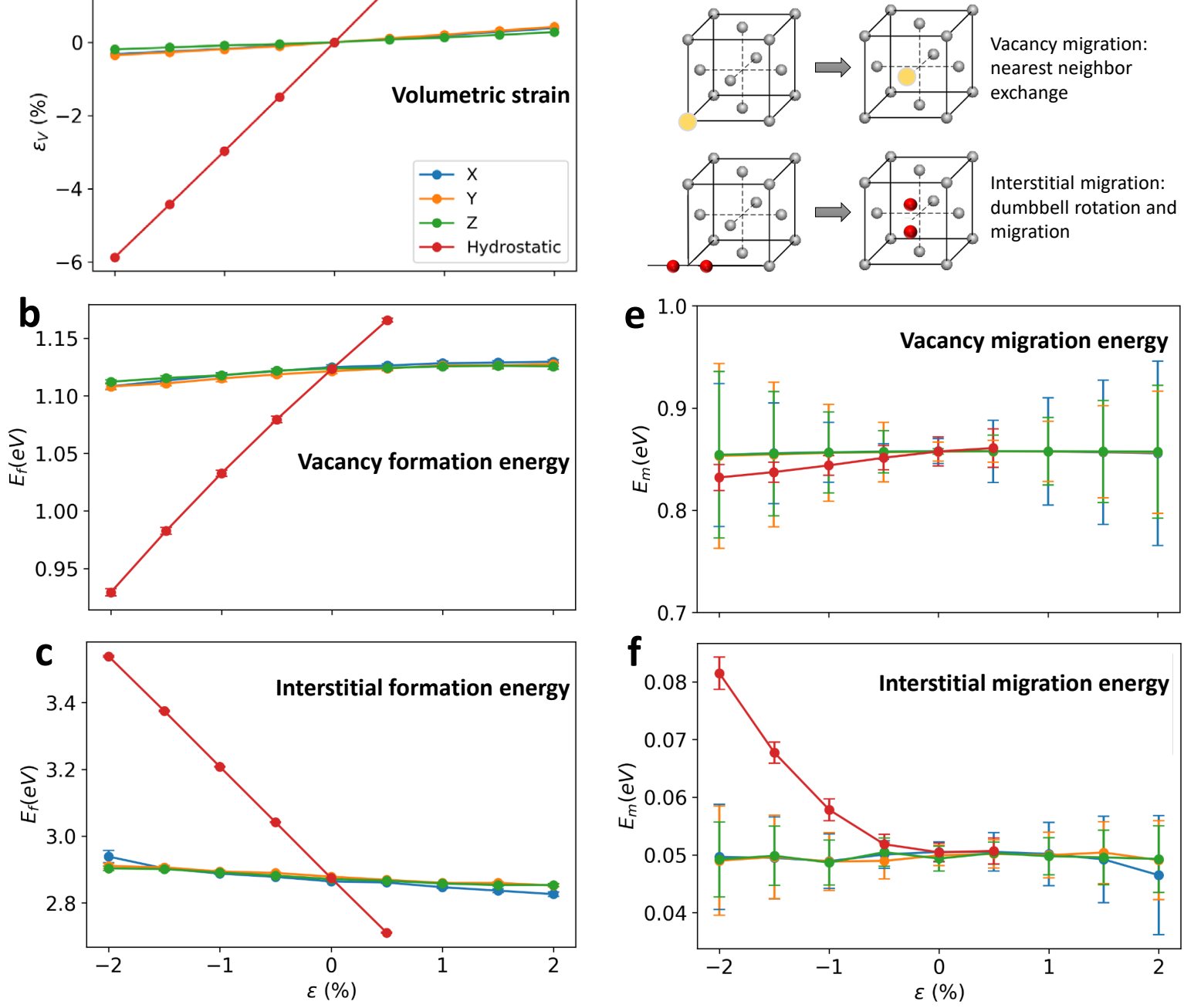

FIG. 7. (a) Volumetric strain, (b) Cu vacancy formation energy, (c) interstitial formation energy,

(d) point defect migration pattern for barrier calculation (yellow: vacancy sites, red: dumbbells),

(e) vacancy migration energy, (f) interstitial migration energy for different strain states. ' $\mathrm{X}$ ', ' $\mathrm{Y}$ ', 'Z', and 'Hydrostatic' stand for uni-axial x, y, z, and hydrostatic strain, respectively. The legend is shown in (a). Error bars denote one standard deviation in calculations considering the directional dependence of defect itself (i.e. interstitial) and migration path.

simulation time scale is limited on the order of tens of nanoseconds, diffusion of vacancies and defect recombination are readily feasible due to the thermally activated events triggered by heat spikes and overlap of cascade-affected volume with pre-existing defects. Reflected in FIG. 7e, vacancy diffusion is very anisotropic, varying significantly between out-of-plane and in-plane directions with respect to the horizontal plane under uni-axial stress. For example, 
under compression $2.0 \%$ in y direction, out-of-plane migration has a high barrier of $0.98 \mathrm{eV}$ while in-plane barrier can be as low as $0.78 \mathrm{eV}$ which facilitates more vacancies to migrate towards the interfaces, but under tension, the behavior swaps leading to more clustering in the bulk (FIG. 5b). This argument applies to x- and z- direction loading as well. Overall, anisotropic diffusion of vacancy can explain well the uni-axial conditions (FIG. 4 and 5). For interstitial diffusion, the barriers are generally much smaller than that of vacancies. Hence, interstitials are expected to be always preferentially lost to sinks, which is consistent with the observation that few interstitials are left in the bulk. By contrast, barriers in the hydrostatic case have a much smaller deviation due to the symmetry in deformation. Take hydrostatic compression as an example: not only defect production is suppressed due to the high Frenkel pair formation energy, but also high interstitial barrier may boost defect recombination, which results in a lower vacancy density (FIG. 4h).

Ion beam mixing of $\mathrm{Zr}$ atoms is dominated by the ballistic and thermal spike phases of the cascades, where $\mathrm{Zr}$ atoms in the AIFs are driven to the bulk. It may lead to the structural instability and precipitation. Previous efforts analyzed the fundamental process of atomic jumps via thermally activated events, and experimentally verified that mixing may be described by the diffusion process $[26,30]$. The distribution variance is a function of chemical properties including mixing energy and cohesive energy. Here, the mixing rate deduced from the diffusion model show consistent linearity with respect to dose, and strain effect does not reveal apparent influence. The underlying reason can be that strain minimally modified the process of ballistic collision and the 'melted' core volume during thermal spike.

\section{CONCLUSION}

With MD simulations of radiation damage on a amorphous-crystalline $\mathrm{Cu}-\mathrm{Zr}$ laminate under various strain conditions including uni-axial and hydrostatic strains, we focus on revealing the correlation between radiation response and strain condition. An overlap of cascades and interfaces reveals that the high sink efficiency of AIFs is hardly reduced due to strain. By introducing numerous consecutive cascades to mimic continuous radiation conditions, interstitials are found preferentially lost at sinks. The saturated vacancy density in the crystalline bulk region demonstrates a strong dependence on strain, in which extension (contraction) of dimension in the direction perpendicular to AIFs shows an increase 
(decrease) in vacancy density. The strain induced vacancy migration anisotropy and point defect formation energy are quantified, which can explain well the observed defect density and size distribution under different stress conditions, stemming from the competing processes of defect production, clustering, recombination, and sinking at AIFs. The underlying defect dynamics has apparently been affected by Zr atom mixing due to modified potential energy landscape, nevertheless, the mixing rate is not obviously influenced by strain.

\section{DECLARATION}

The authors declare that they have no conflict of interest.

[1] G. S. Was, Fundamentals of radiation materials science: metals and alloys (Springer, 2016).

[2] D. Kaoumi, A. Motta, and R. Birtcher, Journal of Applied Physics 104, 073525 (2008).

[3] M. Jin, P. Cao, and M. P. Short, Scripta Materialia 163, 66 (2019).

[4] A. Khalajhedayati, Z. Pan, and T. J. Rupert, Nature Communications 7, 10802 (2016).

[5] Y. Wang, J. Li, A. V. Hamza, and T. W. Barbee, Proceedings of the National Academy of Sciences 104, 11155 (2007).

[6] P. Dubuisson, A. Maillard, C. Delalande, D. Gilbon, and J. Seran, American Society for Testing and Materials, Philadelphia, PA pp. 995-1014 (1992).

[7] K. Kasama, F. Toyokawa, M. Tsukiji, M. Sakamoto, and K. Kobayashi, IEEE Transactions on Nuclear Science 33, 1210 (1986).

[8] C. Xu and G. S. Was, Journal of Nuclear Materials 454, 255 (2014).

[9] M. Cui, N. Gao, D. Wang, X. Gao, and Z. Wang, Nuclear Instruments and Methods in Physics Research Section B: Beam Interactions with Materials and Atoms (2019).

[10] A. Brailsford and R. Bullough, Journal of Nuclear Materials 48, 87 (1973).

[11] B. Beeler, M. Asta, P. Hosemann, and N. Grønbech-Jensen, Journal of Nuclear Materials 459, 159 (2015).

[12] S. Miyashiro, S. Fujita, and T. Okita, Journal of Nuclear Materials 415, 1 (2011).

[13] F. Gao, D. Bacon, P. Flewitt, and T. Lewis, Nuclear Instruments and Methods in Physics Research Section B: Beam Interactions with Materials and Atoms 180, 187 (2001). 
[14] S. Di, Z. Yao, M. R. Daymond, and F. Gao, Nuclear Instruments and Methods in Physics Research Section B: Beam Interactions with Materials and Atoms 303, 95 (2013).

[15] B. Beeler, M. Asta, P. Hosemann, and N. Grønbech-Jensen, Journal of Nuclear Materials 474, $113(2016)$.

[16] M. J. Banisalman and T. Oda, Computational Materials Science 158, 346 (2019).

[17] N. Gao, W. Setyawan, R. J. Kurtz, and Z. Wang, Journal of Nuclear Materials 493, 62 (2017).

[18] C. Kang, Q. Wang, and L. Shao, Journal of Nuclear Materials 485, 159 (2017).

[19] S. Plimpton, P. Crozier, and A. Thompson, Journal of Computational Physics 18 (2007).

[20] V. Borovikov, M. I. Mendelev, and A. H. King, Modelling and Simulation in Materials Science and Engineering 24, 085017 (2016).

[21] J. F. Ziegler, J. P. Biersack, and U. Littmark, The Stopping and Range of Ions in Solids (Pergamon Press, 1985).

[22] K. Nordlund, M. Ghaly, R. Averback, M. Caturla, T. D. de La Rubia, and J. Tarus, Physics Review B 57, 7556 (1998).

[23] A. Stukowski, Modelling and Simulation in Materials Science and Engineering 18, 015012 (2009).

[24] G. Henkelman, B. P. Uberuaga, and H. Jónsson, The Journal of Chemical Physics 113, 9901 (2000)

[25] S. J. Dillon, M. Tang, W. C. Carter, and M. P. Harmer, Acta Materialia 55, 6208 (2007).

[26] R. Averback, Nuclear Instruments and Methods in Physics Research Section B: Beam Interactions with Materials and Atoms 15, 675 (1986).

[27] H. A. Atwater, C. V. Thompson, and H. I. Smith, Journal of Applied Physics 64, 2337 (1988).

[28] P. Shewmon, Diffusion in solids (Springer, 2016).

[29] D. Wang, N. Gao, Z. Wang, X. Gao, W. He, M. Cui, L. Pang, and Y. Zhu, Nuclear Instruments and Methods in Physics Research section B: Beam Interactions with Materials and Atoms 384, 68 (2016).

[30] W. Johnson, Y. Cheng, M. Van Rossum, and M. Nicolet, Nuclear Instruments and Methods in Physics Research Section B: Beam Interactions with Materials and Atoms 7, 657 (1985). 